\title{
Canonical Transformations in a Higher-Derivative Field Theory
}

\author{
L. V. Belvedere, R. L. P. G. Amaral \\ Instituto de Física - Universidade Federal Fluminense \\ Outeiro de São João Batista s/n, 24020-005 Centro, Niterói, Rio de Janeiro - Brazil \\ N. A. Lemos* \\ Center for Theoretical Physics \\ Laboratory for Nuclear Science and Department of Physics \\ Massachusetts Institute of Technology \\ Cambridge, MA 02139 - USA
}

August 4, 2018

\begin{abstract}
It has been suggested that the chiral symmetry can be implemented only in classical Lagrangians containing higher covariant derivatives of odd order. Contrary to this belief, it is shown that one can construct an exactly soluble two-dimensional higher-derivative fermionic quantum field theory containing only derivatives of even order whose classical Lagrangian exhibits chiral-gauge invariance. The original field solution is expressed in terms of usual Dirac spinors through a canonical transformation, whose generating function allows the determination of the new Hamiltonian. It is emphasized that the original and transformed Hamiltonians are different because the mapping from the old to the new canonical variables depends explicitly on time. The violation of cluster decomposition is discussed and the general Wightman functions satisfying the positive-definiteness condition are obtained.
\end{abstract}

PACS numbers: 11.10.Ef, 11.15.Tk

${ }^{*}$ On leave of absence from Departamento de Física, Universidade Federal Fluminense, Outeiro de São João Batista s/n, 24020-005 Centro, Niterói, RJ, Brazil. 


\section{Introduction}

In the last few years several authors have turned their attention to the study of higher-derivative field theories. The reader is referred to [1-3] for the motivation of such investigations, as well as for a brief sketch of the historical development of the subject. It is the purpose of this paper to show that certain statements concerning higher-derivative field theories with chiral symmetry are based on hasty arguments, and to make the discussion concrete a two-dimensional higher-derivative free model of even order and its local gauge invariant generalization (higher-derivative Schwinger model of even-order) are considered. We also address ourselves to some unexplored aspects of two-dimensional quantum field theories with higher-derivative couplings.

One occasionally finds in the literature statements to the effect that the chiral symmetry restricts "the number and the kind" of covariant derivatives in the fermionic Lagrangian, so that, for instance, only the appearence of an odd number of covariant derivatives of the fermion fields is compatible with invariance (at the classical level) of the fermionic Lagrangian under chiralgauge transformations [3]. Here we show that this is not the case by explicitly constructing a higher-derivative model of even order whose classical Lagrangian exhibits chiral-gauge invariance.

The local operator solution of this higher-derivative model is obtained in terms of a product of local spacetime functions and usual "bona fide" Dirac spinors, thus defining a mapping between two sets of field operators. It is pointed out that, being explicitly time dependent, such a mapping leads to a new Hamiltonian $H^{\prime}$ that differs from the original Hamiltonian $H$. The canonical character of the transformation is stressed and the generating function involving old and new variables is obtained. The same analysis can be extended to higher-derivative models of odd order.

The theory involves an indefinite-metric "Hilbert space" and cluster decomposition is violated. It is shown, however, that the general Wightman functions for the canonical free and massless Dirac fields satisfying the positive-definiteness condition can be recovered by considering correlations between the original field variables and their conjugate momenta.

\section{Free Model}

Attention has been called recently $[2,3]$ to Lorentz invariant field models in two-dimensional spacetime defined by the Lagrangian density

$$
\mathcal{L}_{o}=i \bar{\xi}\left(\not \partial \partial^{\dagger}\right)^{N} \not \partial \xi,
$$


whose local gauge-invariant generalization can be exactly solved円.

With the purpose of introducing the extension to models with an even number of field derivatives, let us consider the Lorentz transformation (L T) properties in two dimensions. In this case, the behavior of fields under a $\mathrm{L} \mathrm{T}$ is better analyzed using the light-cone variables : $x^{ \pm}=x^{0} \pm x^{1}$. Under a L T these variables transform according with $x^{+} \rightarrow \lambda x^{+}, x^{-} \rightarrow \lambda^{-1} x^{-}$, such that $\partial_{+} \rightarrow \lambda^{-1} \partial_{+}$and $\partial_{-} \rightarrow \lambda \partial_{-}$, where $\lambda \in(0, \infty)$. In terms of spinor components $\xi_{(\alpha)}$, the kinetic term (1.1) can be written as

$$
\mathcal{L}_{o}=i \xi_{(1)}^{*} \partial_{-}^{2 N+1} \xi_{(1)}+i \xi_{(2)}^{*} \partial_{+}^{2 N+1} \xi_{(2)}
$$

Thus, under a L T the upper and lower spinor components obey the transformation law $\xi_{(\alpha)} \rightarrow \lambda^{\left(N+\frac{1}{2}\right) \gamma_{\alpha \alpha}^{5}} \xi_{(\alpha)}$.

Taking these considerations into account, the extension to the case with an even number of Fermi fields derivatives can be introduced through the following (Hermitian) Lagrangian density

$$
\mathcal{L}_{o}=\bar{\xi} \gamma^{0}\left(i \not \partial^{\dagger} i \not \partial\right)^{N} \xi
$$

where the imaginary factor $i$ was inserted for future convenience. In this case, the Lorentz transformation properties of the spinor components are $\xi_{(\alpha)} \rightarrow \lambda^{-N \gamma_{\alpha \alpha}^{5}} \xi_{(\alpha)}$.

Let us now perform the quantization of the free model defined by the Lagrangian (1.3). For the sake of simplicity we consider the upper component only. Proceeding in the spirit of Ref. [2], we consider the configuration space generated by

$$
\xi_{(1)}^{n}=\left(\partial_{-}\right)^{n} \xi_{(1)}, n=0,1, . ., 2 N-1
$$

so that the associated momenta are given by

$$
\pi_{\xi}^{n}=(-1)^{(N-1-n)}\left(\partial_{-}\right)^{(2 N-1-n)} \xi_{(1)}^{*}
$$

Since the system under consideration exhibits constraints, the canonical quantization must be per-

$$
\begin{gathered}
{ }^{1} \text { The conventions used are }: \xi=\left(\xi_{(1)}, \xi_{(2)}\right)^{T}, \epsilon^{01}=g^{00}=-g^{11}=1, \\
\gamma^{5}=-\gamma^{0} \gamma^{1}, \gamma^{\mu} \gamma^{5}=\epsilon^{\mu \nu} \gamma_{\nu} \\
\gamma^{0}=\left(\begin{array}{ll}
0 & 1 \\
1 & 0
\end{array}\right), \gamma^{1}=\left(\begin{array}{cc}
0 & 1 \\
-1 & 0
\end{array}\right), \gamma^{5}=\left(\begin{array}{cc}
1 & 0 \\
0 & -1
\end{array}\right)
\end{gathered}
$$


formed using the Dirac method, which leads to the equal-time anticommutators

$$
\left\{\partial_{-}^{p} \xi_{(1)}(x), \partial_{-}^{q} \xi_{(1)}^{*}(y)\right\}_{E T}=i(-1)^{N-p-1} \delta_{q+p, 2 N-1} \delta\left(x^{1}-y^{1}\right)
$$

In momentum space, the solution of the equation of motion for $\xi_{(1)}$ is a linear combination of derivatives of $\delta\left(k^{2}\right)$ up to the order $(N-1)$. The Fourier representation for the operator solution of the model which leads to a local field operator is given by $\left(k \equiv k_{+}\right)$

$$
\xi_{(1)}(x)=\frac{m^{\frac{1}{2}-N}}{\sqrt{2 \pi}} \int_{-\infty}^{+\infty} d k e^{-i k\left(x^{0}+x^{1}\right)} \sum_{p=0}^{2 N-1} \frac{\left(-i m x^{-}\right)^{p}}{p !} \tilde{\xi}_{(1)}^{p}(k)
$$

In the above expression the finite arbitrary mass scale $m$ is introduced $\mathbb{f}^{2}$ in order to ensure the usual dimension for the spinor component fields $\tilde{\xi}_{(1)}^{p}(k)$, which satisfy

$$
\left\{\tilde{\xi}_{(1)}^{p}(k), \tilde{\xi}_{(1)}^{q *}\left(k^{\prime}\right)\right\}=\delta_{q+p, 2 N-1} \delta\left(k-k^{\prime}\right)
$$

The anticommutation relations of the mode expansion operators can be diagonalized via the linear transformation

$$
\begin{gathered}
\tilde{\psi}_{(1)}^{p}(k)=\frac{1}{\sqrt{2}}\left(\tilde{\xi}_{(1)}^{p-1}(k)+\tilde{\xi}_{(1)}^{2 N-p}(k)\right), \\
\tilde{\psi}_{(1)}^{N+p}(k)=\frac{1}{\sqrt{2}}\left(\tilde{\xi}_{(1)}^{p-1}(k)-\tilde{\xi}_{(1)}^{2 N-p}(k)\right),
\end{gathered}
$$

with $p=1,2, . ., N$. Now a set of $2 N$ usual free Dirac spinors in coordinate space $\psi^{j}$ can be introduced. Defining their upper components by

$$
\psi_{(1)}^{p}(x)=\frac{1}{\sqrt{2 \pi}} \int d k e^{-k \cdot\left(x^{0}+x^{1}\right)} \tilde{\psi}_{(1)}^{p}(k)
$$

we can write

$$
\xi_{(1)}(x)=\sum_{j=1}^{2 N} f_{j}\left(x^{-}\right) \psi_{(1)}^{j}\left(x^{+}\right)
$$

\footnotetext{
${ }^{2}$ It should be remarked that there is another solution, in which the arbitrary dimensional parameter $m$ is not introduced, but it leads to a non-local field operator. In order to circumvent this problem and obtain a solution in terms of usual fermionic field operators, the parameter $m$ must be introduced. In this case, the previously referred Lorentz transformation properties of the spinor components $\xi_{(\alpha)}$ are implemented if we perform the LT combined with the redefinition $m \rightarrow \lambda m$.
} 
The Dirac fields $\psi_{(1)}^{j}\left(x^{+}\right)$are quantized with positive (negative) metric for $j \leq N(j>N)$. The factors $f_{j}\left(x^{-}\right)$are given by

$$
\begin{gathered}
f_{j}\left(x^{-}\right)=\frac{m^{\frac{1}{2}-N}}{\sqrt{2}}\left[\frac{\left(-i m x^{-}\right)^{j-1}}{(j-1) !}+\frac{\left(-i m x^{-}\right)^{2 N-j}}{(2 N-j) !}\right] \\
f_{j+N}\left(x^{-}\right)=(-1)^{j+1} f_{j}^{*}\left(x^{-}\right)
\end{gathered}
$$

where $j \leq N$. The expression for the lower component $\xi_{(2)}(x)$ is obtained interchanging the lightcone variables $x^{+} \leftrightarrow x^{-}$. In order to ensure the correct Lorentz transformation properties of the lower spinor component, a new dimensional parameter $\tilde{m}$ must be introduced such that under a Lorentz transformation it is redefined as $\tilde{m} \rightarrow \lambda^{-1} \tilde{m}$. In this way, the higher-order "spinor field" can be expressed as a linear combination of usual Dirac fields.

The anticommutator between $\xi_{(\alpha)}(\alpha=1,2)$ fields is given by

$$
\left\{\xi_{(\alpha)}^{*}(x), \xi_{(\alpha)}(y)\right\}=\frac{(-i)^{2 N-1}}{(2 N-1) !}\left(x^{\mp}-y^{\mp}\right)^{2 N-1} S\left(x^{ \pm}-y^{ \pm}\right)
$$

where $S(x)$ is the anticommutator of two usual free and massless Dirac fields. Although the $\xi$ field does not represent a genuine spinor field operator, the corresponding spinorial nature is carried by the infrafermion $\psi^{j}$ field operators, which ensure the correct micro-causality requirements. In this sense, the general quantum field features are implemented by the infrafermion operators.

As expected from a theory with an indefinite-metric "Hilbert space", the cluster decomposition is violated. As a consequence of (1.13), for $\alpha=1(2)$ the $2 p$-point functions of the $\xi_{(\alpha)}$ fields violate the cluster decomposition in the light-cone variable $x^{-}\left(x^{+}\right)$. Nevertheless, the general Wightman functions generated by the canonical free and massless Dirac spinor components $\psi_{(\alpha)}^{o}\left(x^{ \pm}\right)$satisfying the positive-definiteness condition can be recovered considering arbitrary correlations between the configuration space variable $\xi^{\ell}(x)$ and the associated momenta $\pi_{\xi}^{\ell}(x)$ defined respectively by Eqs.(1.4) and (1.5). Thus, for the $2 p$-point function with fixed $\ell=0, \ldots, 2 N-1$, we get

$$
\begin{gathered}
\left\langle 0\left|\prod_{j=1}^{p} \pi_{\xi_{(\alpha)}}^{\ell}\left(x_{j}\right) \prod_{k=1}^{p} \xi_{(\alpha)}^{\ell}\left(y_{k}\right)\right| 0\right\rangle=\left\langle 0\left|\prod_{j=1}^{p}\left(\partial_{\mp}\right)^{2 N-1-\ell} \xi_{(\alpha)}^{*}\left(x_{j}\right) \prod_{k=1}^{p}\left(\partial_{\mp}\right)^{\ell} \xi_{(\alpha)}\left(y_{k}\right)\right| 0\right\rangle \equiv \\
\quad \equiv c\left\langle 0\left|\prod_{j=1}^{p} \psi_{(\alpha)}^{o *}\left(x_{j}^{ \pm}\right) \prod_{k=1}^{p} \psi_{(\alpha)}^{o}\left(y_{k}^{ \pm}\right)\right| 0\right\rangle=c \frac{\prod_{i<j}\left(x_{i}-x_{j}\right) \prod_{k<m}\left(y_{k}-y_{m}\right)}{\prod_{i \neq k}\left(x_{i}-y_{k}\right)}
\end{gathered}
$$

where $c$ is a constant. 
The bosonization of the free theory can be carried out using the stardard bosonization formulae, leading to $N$ independent $\phi_{j}$ scalar fields associated to the Dirac spinor $\psi^{j}$. Similarly to what is done in Refs.[2,4], we introduce the following decomposition for the $2 N$ independent scalar fields :

$$
\phi_{j}=\frac{1}{\sqrt{2 N}} \Phi+\sum_{i_{D}=1}^{2 N-1} \lambda_{j j}^{i_{D}} \varphi^{i_{D}}
$$

where $\lambda_{j j}^{i_{D}}$ are the diagonal matrices of the $\mathcal{S U}(2 N)$. The $\Phi$ field acts as the potential for $\mathcal{U}(1)$ and chiral- $\mathcal{U}(1)$ conserved currents. Using this decomposition in the usual bosonized expression for the free Dirac fermion operator, the $\mathcal{U}(1)$ and chiral- $\mathcal{U}(1)$ dependence factorize and we get

$$
\xi_{(1)}(x)=S_{(1)}^{\Phi}\left(x^{+}\right) \sum_{j=1}^{2 N} f_{j}\left(x_{-}\right) \hat{\psi}_{(1)}^{j}\left(x^{+}\right),
$$

where the "soliton" operator $S^{\Phi}$ and the $\mathcal{U}(1)$ screened Dirac infrafermions $\hat{\psi}^{j}$ are given by [4]

$$
\begin{gathered}
S_{(1)}^{\Phi}\left(x^{+}\right)=: \exp \left\{2 i \sqrt{\frac{\pi}{2 N}} \Phi\left(x^{+}\right)\right\}: \\
\hat{\psi}_{(1)}^{j}\left(x^{+}\right)=\left(\frac{\mu}{2 \pi}\right)^{\frac{1}{2}}: \exp \left\{2 i \sqrt{\pi} \sum_{i_{D}=1}^{2 N-1} \lambda_{j j}^{i_{D}} \varphi^{i_{D}}\left(x^{+}\right)\right\}: .
\end{gathered}
$$

In the expressions above we have suppressed the corresponding Klein factors needed to ensure the correct anticommutation relations [5]. As stressed in [2], these Klein factors are also introduced in order to implement the indefinite metric Hilbert space.

\section{Canonical Transformation}

In this section we shall obtain the Hamiltonian $H$ for the model defined by the Lagrangian density (1.3), which evolves the $\xi$ field, and show that it is related by a canonical transformation to the Hamiltonian $H^{\prime}$ that describes the time evolution of the usual Fermi field $\psi$. For the sake of simplicity we consider the upper component only.

To begin with we use a generalized Legendre transformation of the Lagrangian density (1.3), such that the Hamiltonian density $\mathcal{H}$ can be written as

$$
\mathcal{H}(x)=\sum_{n=0}^{2 N-1} \pi_{\xi}^{n}(x) \dot{\xi}_{(1)}^{n}(x)-\xi_{(1)}^{*}(x)\left(i \partial_{-}\right)^{2 N} \xi_{(1)}(x)
$$

Using the definitions (1.4) for the configuration space field variables and (1.5) for the corresponding associated momenta, we obtain the Hamiltonian density (2.1a) in terms of the phase-space 
variables as being

$$
\mathcal{H}(x)=\sum_{n=0}^{2 N-2}(-1)^{N-1-n} \xi_{(1)}^{* 2 N-1-n} \xi_{(1)}^{n+1}(x)+\sum_{n=0}^{2 N-1}(-1)^{N-1-n} \xi_{(1)}^{* 2 N-1-n}(x) \partial_{1} \xi_{(1)}^{n}(x) .
$$

The corresponding Hamiltonian $H$ evolves the field $\xi_{(1)}(x)$. Since the expression of the original configuration space variables in terms of the $2 N$ usual Dirac fields $\psi^{j}$ is explicitly time dependent, the Hamiltonian obtained from (2.1) does not describe the time evolution of the latter fields. Nevertheless let us show that the Hamiltonian $H^{\prime}$ that describes the time evolution of the Dirac fields can be obtained from $H$ by canonical methods.

To this end, we consider Eq.(1.11) as defining a mapping from the set of old variables $\xi_{(1)}^{p}$, given by Eq.(1.4), to the set of new variables $\psi_{(1)}^{p}$,

$$
\left\{\xi_{(1)}^{n}(x)\right\} \rightarrow\left\{\psi_{(1)}^{p}(x)\right\}
$$

Taking into account the expressions for all the original phase space variables given by (1.11), we have the set of transformation equations:

$$
\xi_{(1)}^{n}(x)=\sum_{j=1}^{2 N}\left[\partial_{-}^{n} f_{j}\left(x^{-}\right)\right] \psi_{(1)}^{j}(x)
$$

After this point transformation, the new momenta are obtained computing the variation of the action around a solution of the equation of motion:

$$
\delta S=\int_{t_{1}}^{t_{2}} d^{2} x \xi_{(1)}^{*}(x)\left(i \partial_{-}\right)^{2 N} \delta \xi_{(1)}(x)
$$

Considering vanishing variations at the initial time $t_{1}$, we get

$$
\delta S=i \sum_{j=1}^{N}\left[\psi_{(1)}^{* j}(x) \delta \psi_{(1)}^{j}(x)-\psi_{(1)}^{*(j+N)}(x) \delta \psi_{(1)}^{j+N}(x)\right]_{t=t_{2}} .
$$

and we can identify $i \psi_{(1)}^{* j}$ as the canonical conjugate momenta of the $\psi_{(1)}^{j}$ fields. The anticommutators for the Dirac fields are thus reobtained without resort to the anticommutation relations of the original field solution.

In order to obtain the expression of the new Hamiltonian, let us consider the generating function for the point transformation (2.3). In classical mechanics the generating function of a point transformation from the set of conjugate variables $\left\{p_{i}, q_{i}\right\}$ to the set $\left\{P_{i}, Q_{i}\right\}$ can be written as

$$
\Omega(P, q, t)=\sum_{i} P_{i} Q_{i}(q, t)
$$


The transformation equations can be recovered by means of

$$
Q_{i}=\frac{\partial \Omega}{\partial P_{i}} \quad, \quad p_{i}=\frac{\partial \Omega}{\partial q_{i}}
$$

while the expression for the new Hamiltonian function is given by

$$
\mathcal{H}^{\prime}=\mathcal{H}+\partial_{o} \Omega
$$

The generalization of this formalism to higher-derivative field theory can be achieved by considering the "generating function" variables as the sum of the old momenta multiplied by the corresponding old variables written in terms of the new ones, that is,

$$
\Omega_{(1)}(x)=\sum_{n=0}^{2 N-1}\left\{(-1)^{N-1-n} \xi_{(1)}^{*(2 N-1-n)}(x) \sum_{j=1}^{2 N}\left[\partial_{-}^{n} f_{j}\left(x^{-}\right)\right] \psi_{(1)}^{j}\left(x^{+}\right)\right\} .
$$

This generating function allows one to obtain the transformation (2.3) through

$$
\begin{gathered}
\xi_{(1)}^{n}(x)=(-1)^{N-1-n} \frac{\partial \Omega(x)}{\partial \xi_{(1)}^{* 2 N-n-1}(x)} \\
i(-1)^{\ell} \psi_{(1)}^{j *}=\frac{\partial \Omega(x)}{\partial \psi_{(1)}^{j}(x)}
\end{gathered}
$$

where $\ell=0(1)$, for $j \leq N(j>N)$. The new Hamiltoninan density $\mathcal{H}^{\prime}$ differing from $\mathcal{H}$ by $\partial_{o} \Omega_{(1)}(x)$ is given by

$$
\mathcal{H}^{\prime}=\sum_{j=1}^{N}\left[i \psi_{(1)}^{* j}(x) \partial_{1} \psi_{(1)}^{j}(x)-i \psi_{(1)}^{* j+N}(x) \partial_{1} \psi_{(1)}^{j+N}(x)\right]
$$

which is the correct Hamiltonian density associated with $N$ independent free Dirac spinor components $\psi_{(1)}^{j}(x)$, and the one to be bosonized.

Analogous expressions are obtained for the lower component $\psi_{(2)}^{j}(x)$.

\footnotetext{
3 Strictly speaking one should consider a generating functional obtained by integrating $\Omega_{(1)}(x)$ over space. In the peculiar case dealt with here this is not necesssary, and the formalism with generating function works quite satisfactorily.
} 


\section{Local Gauge Invariance}

The Schwinger model with derivatives of order $2 N$ is defined by the Lagrangian density

$$
\mathcal{L}=-\frac{1}{4}\left(\mathcal{F}_{\mu \nu}\right)^{2}+\bar{\Psi} \not^{2 N} \Psi
$$

where $4^{2 N}$ is the covariant derivative of order $2 N$ defined by

$$
{\chi^{2 N}}^{N}=\gamma^{0}\left(i \not^{\dagger} i \not \mathcal{D}\right)^{N}
$$

where the usual covariant derivative is $\not{D}=\gamma^{\mu}\left(\partial_{\mu}-i\right.$ e $\left.\mathcal{A}_{\mu}\right)$.

The quantization of the model can be performed following the same steps outlined in the freefield case. The configuration space is now generated by

$$
\Psi^{n}=\mathcal{D}_{ \pm}^{n} \Psi \quad n=0,1,2, \ldots, 2 N-1
$$

and the associated momenta

$$
\pi_{\Psi}^{n}=(-1)^{(N-1-n)}\left(\mathcal{D}^{2 N-1-n} \Psi\right)^{*}
$$

which satisfy canonical anticommutation relations. Since a chiral operator gauge transformation acting on the free field decouples the gauge field, similarly to what is done in Ref.[2], the configuration space variables can be written as

$$
\begin{gathered}
\Psi^{n}(x)=: e^{i \sqrt{\frac{\pi}{2 N}} \gamma^{5}[\tilde{\Sigma}(x)+\tilde{\eta}(x)]}: \xi^{n}(x), \\
\pi_{\Psi}^{n}(x)=: e^{-i \sqrt{\frac{\pi}{2 N}} \gamma^{5}[\tilde{\Sigma}(x)+\tilde{\eta}(x)]}: \pi_{\xi}^{n}(x) .
\end{gathered}
$$

The operator solution for the even order case is then given by

$$
\Psi_{(\alpha)}(x)=: \exp \left\{i\left(\frac{\pi}{2 N}\right)^{\frac{1}{2}} \gamma_{\alpha \alpha}^{5}[\tilde{\Sigma}(x)+\tilde{\eta}(x)]\right\}: \sum_{j=1}^{2 N} f_{j}\left(x^{\mp}\right) \psi^{j}\left(x^{ \pm}\right)
$$


Performing an operator gauge transformation

$$
\hat{\Psi}_{(\alpha)}(x)=: e^{i \sqrt{\frac{\pi}{2 N}} \eta(x)}: \Psi_{(\alpha)}(x),
$$

we obtain the "physical fermion field operator"

$$
\hat{\Psi}_{(\alpha)}(x)=: \exp \left\{i\left(\frac{\pi}{2 N}\right)^{\frac{1}{2}} \gamma_{\alpha \alpha} \tilde{\Sigma}(x)\right\}: \sum_{j=1}^{2 N} f_{j}\left(x^{\mp}\right) \hat{\psi}^{j}\left(x^{ \pm}\right) \sigma
$$

where $\sigma$ is a spurious operator with scale dimension of zero value and given in terms of free and massless scalar fields by

$$
\sigma=\exp \left\{i\left(\frac{\pi}{2 N}\right)^{\frac{1}{2}}\left\{\gamma^{5}[\tilde{\Phi}(x)+\tilde{\eta}(x)]+[\Phi(x)+\eta(x)]\right\}\right\}
$$

where the $\eta$ field is quantized with negative metric. The gauge field is given by

$$
\hat{\mathcal{A}}_{\mu}(x)=-\frac{1}{e} \sqrt{\frac{\pi}{2 N}} \varepsilon_{\mu \nu} \partial^{\nu} \tilde{\Sigma}(x)
$$

with $\tilde{\Sigma}$ satisfying the equation of motion

$$
\left[\square+\frac{2 N e^{2}}{\pi}\right] \tilde{\Sigma}(x)=0
$$

and the anomalous divergence of the axial current is then given by

$$
\partial^{\mu} \Im_{\mu}^{5}=-\frac{N e}{\pi} \varepsilon_{\mu \nu} \mathcal{F}^{\mu \nu}
$$

The generalized fermion Green functions are obtained considering correlators like

$$
\begin{aligned}
& \left\langle 0\left|\prod_{j=1}^{p}\left(\mathcal{D}_{\mp}^{2 N-1-\ell} \Psi_{(\alpha)}\left(x_{j}\right)\right)^{*} \prod_{k=1}^{p} \mathcal{D}_{\mp}^{\ell} \Psi_{(\alpha)}\left(y_{k}\right)\right| 0\right\rangle=\left\langle 0\left|\prod_{j=1}^{p} \psi_{(\alpha)}^{o *}\left(x_{j}^{ \pm}\right) \prod_{k=1}^{p} \psi_{(\alpha)}^{o}\left(y_{k}^{ \pm}\right)\right| 0\right\rangle \times \\
& \times\left\langle 0\left|\prod_{j=1}^{p}: e^{-i \sqrt{\frac{\pi}{2 N}} \gamma_{\alpha \alpha}^{5}\left[\tilde{\Sigma}\left(x_{j}\right)+\tilde{\eta}\left(x_{j}\right)\right]}: \prod_{k=1}^{p}: e^{i \sqrt{\frac{\pi}{2 N}} \gamma_{\alpha \alpha}^{5}\left[\tilde{\Sigma}\left(y_{k}\right)+\tilde{\eta}\left(y_{k}\right)\right]}:\right| 0\right\rangle .
\end{aligned}
$$


The Wightman functions related to the physical fermion field operators $\hat{\Psi}$, are given by

$$
\begin{gathered}
\left\langle 0\left|\prod_{j=1}^{p}\left(\mathcal{D}_{\mp}^{2 N-1-\ell} \hat{\Psi}_{(\alpha)}\left(x_{j}\right)\right)^{*} \prod_{k=1}^{p} \mathcal{D}_{\mp}^{\ell} \hat{\Psi}_{(\alpha)}\left(y_{k}\right)\right| 0\right\rangle=\left\langle 0\left|\prod_{j=1}^{p} \hat{\psi}_{(\alpha)}^{*}\left(x_{j}^{ \pm}\right) \prod_{k=1}^{p} \hat{\psi}_{(\alpha)}\left(y_{k}^{ \pm}\right)\right| 0\right\rangle \times \\
\quad \times\left\langle 0\left|\prod_{j=1}^{p}: e^{-i \sqrt{\frac{\pi}{2 N}} \gamma_{\alpha \alpha}^{5} \tilde{\Sigma}\left(x_{j}\right)}: \prod_{k=1}^{p}: e^{i \sqrt{\frac{\pi}{2 N}} \gamma_{\alpha \alpha}^{5} \tilde{\Sigma}\left(y_{k}\right)}:\right| 0\right\rangle
\end{gathered}
$$

in which we have used the fact that

$$
\left\langle 0\left|\prod_{j=1}^{p} \sigma_{\alpha}^{*}\left(x_{j}\right) \prod_{k=1}^{p} \sigma_{\alpha}\left(y_{k}\right)\right| 0\right\rangle=1
$$

The Wightman functions (3.15) correspond to those of a subspace of the Schwinger model with $2 N$ flavored fermions introduced in Ref.[4].

\section{Final Comments}

By way of conclusion, let us summarize our findings. We have further extended the class of models amenable to exact solution studied in [2] by considering Lagrangians involving higher derivatives of even order. These Lagrangians are immediately seen to be Lorentz invariant by using light-cone variables. The resulting unusual behavior of the fields in these generalized Schwinger models has been stressed. As a final result the bosonization of models with Lagrangians of the form

$$
L=\xi^{\dagger}\left(i \gamma^{0} \not \partial\right)^{N} \xi, \quad N=1,2,3, \ldots
$$

has been obtained as well as of their gauged versions. Special care, irrespective of $N$ being even or odd, is needed when the bosonization of expressions as the Lagrangian or the Hamiltonian is undertaken. Indeed, by considering the solution of the model as a point transformation of fields the generating function for this transformation has been obtained and therefrom the new Hamiltonian. Owing to explicit spacetime dependence of the original fields in terms of the ultimately bosonized internal fields $\psi^{j}$, the bosonized Hamiltonians of the latter are not obtained by just bosonizing the original Hamiltonians expressed in terms of the new fields. 
Acknowledgment : The authors express their thanks to Conselho Nacional de Desenvolvimento Científico e Tecnológico (CNPq), Brazil, for partial financial support.

\section{References}

1 - C. Batlle, J. Gomis, J. M. Pons and N. Roman-Roy, J.Phys. A21, 2963(1988);

V. V. Nesterenko, ibid A22, 1673(1989);

C. A. P. Galvão and N. A. Lemos, J. Math. Phys. 29, 1588(1988);

J. Barcelos-Neto and N. R. F. Braga, Acta Phys. Polonica 20, 205(1989);

J. Barcelos-Neto and C. P. Natividade, Z. Phys. C49, 511(1991);

ibid C51, 313(1991);

J. J. Giambiagi, Nuovo Cimento 104 A, 1841 (1991);

C. G. Bolini and J. J. Giambiagi, J. Math. Phys. 34, 610 (1993)

2 - R. L. P. G. do Amaral, L. V. Belvedere, N. A. Lemos and C. P. Natividade, Phys. Rev. D47, $3443(1993)$

3 - J. Barcelos-Neto and C. P. Natividade, Z. Phys. C 49, 511 (1991)

4 - L. V. Belvedere, J. A. Swieca, K. D. Rothe and B. Schroer, Nucl. Phys. B 153, 112 (1979);

L. V. Belvedere, Nucl. Phys. B 276, 197 (1986).

5 - M. B. Halpern, Phys. Rev. D 12, 1684 (1975); 13, 337 (1976). 